Remote System and Sensor

Applications (8A800

5. Proj./Prog./Dept./Div.:

LDUA Project

8. Originator Remarks:

Initial release of the Operation and Maintenance Manual for the High Resolution Steroscopic Video Camera System (HRSVS). ETN-96-0007.
3. From: (Originating Organization)

Remote System and Sensor Applications (8A800)

6. Cog. Engr.:

A. F. Pardini
2. To: (Receiving organization)

4. Related EDT Mo.:

N/A

7. Purchase Order No.:

N/A

9. Equip./Component No.:

LDUA/6230

10. Systen/8ldg./Facility: N/A

12. Major Assm. Dwg. No.: N/A

13. Permit/Permit Application Mo.: $N / A$

14. Required Response Date: $N / A$

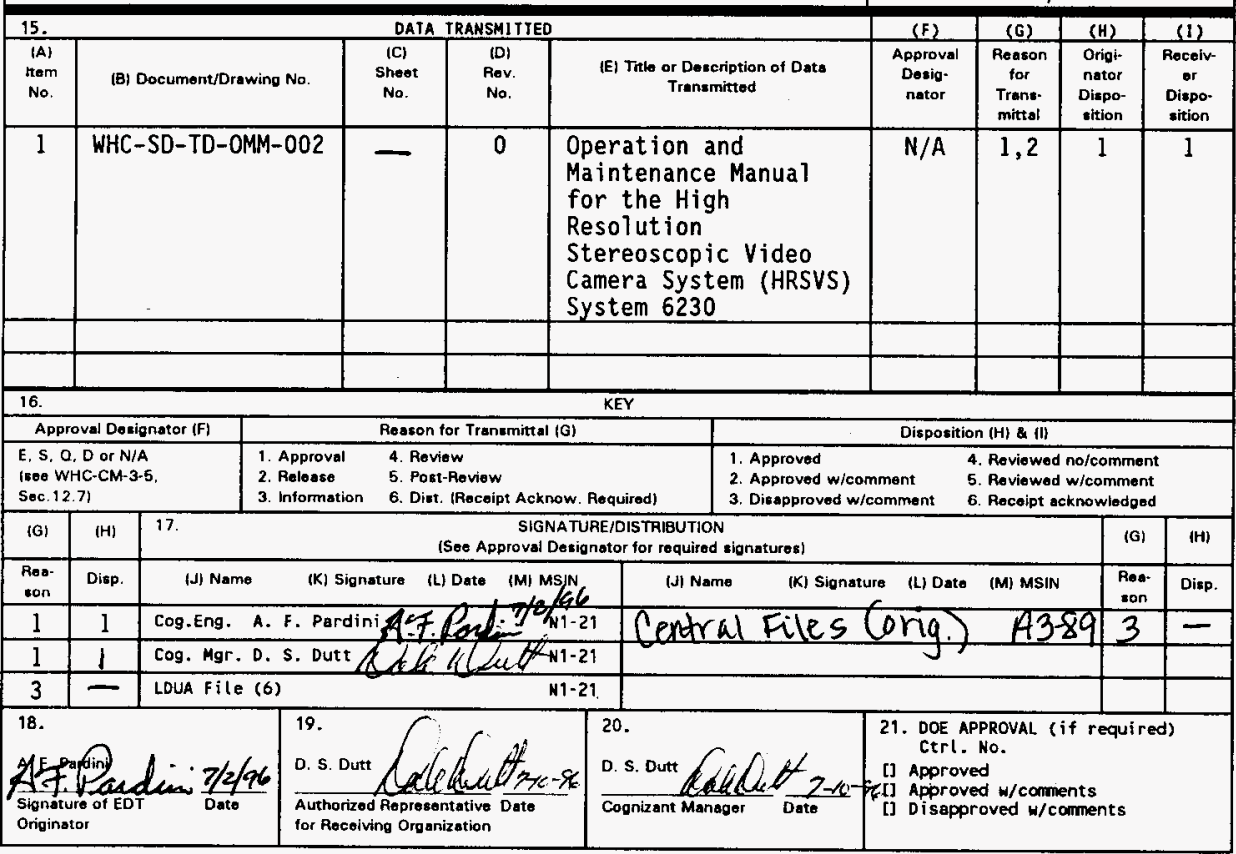

BD-7400-172-2 (04/94) GEF097 


\section{Operation and Maintenance Manual for the High Resolution Stereoscopic Video Camera System (HRSVS) System 6230}

\section{A. F. Pardini}

Westinghouse Hanford Company, Richland, WA 99352

U.S. Department of Energy Contract DE-AC06-87RL10930

$\begin{array}{lll}\text { EDT/ECN: } & 612372 & \text { UC: } 2060 \\ \text { Org Code: } & 84800 & \text { Charge Code: H1E02 } \\ \text { B\&R Code: } & \text { EW4010000 } & \text { Total Pages: } 2529\end{array}$

Key Words: High Resolution Stereoscopic Video Camera System, HRSVS, Light Duty Utility Arm, LDUA, Supervisory Data Acquisition System, SDAS, Operations and Maintenance, $O$ and $M$.

Abstract: The High Resolution Stereoscopic Video Camera System (HRSVS), system 6230, is a stereoscopic camera system that will be used as an end effector on the LDUA to perform surveillance and inspection activities within Hanford waste tanks. It is attached to the LDUA by means of a Tool Interface Plate (TIP), which provides a feed through for all electrical and pneumatic utilities needed by the end effector to operate.

TRADEMARK DISCLAIMER. Reference herein to any specific comercial product, process, or service by trade name, trademark, manufacturer, or otherwise, does not necessarily constitute or imply its endorsement, recommendation, or favoring by the United states Government or any agency thereof or its contractors or subcontractors.

Printed in the United States of America. To obtain copies of this document, contact: BKC/BCS Document Control Services, P.O. BOX 1970, Mailstop H6-08, Richland WA 99352, Phone (509) 372-2420; fax (509) 376-4989.
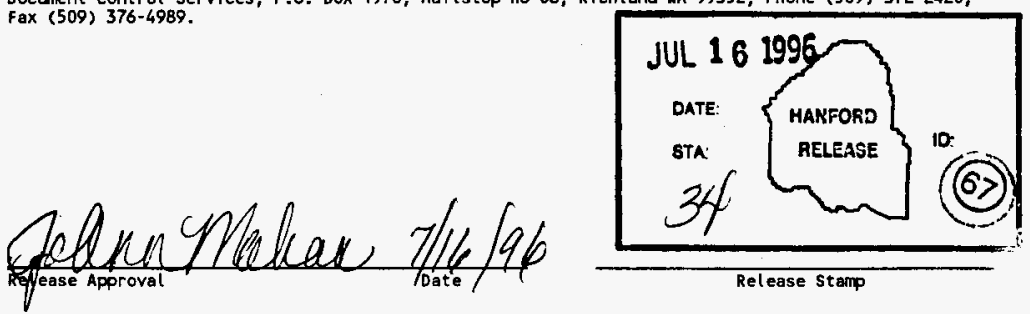

Approved for Public Release 
WHC-SD-TD-OMM-002, REV. 0

OPERATION AND MAINTENANCE MANUAL

FOR THE HIGH RESOLUTION STEREOSCOPIC VIDEO CAMERA SYSTEM

(HRSVS)

SYSTEM 6230

JUNE 27, 1996

BY

A. F. PARDINI

REMOTE SYSTEM AND SENSOR APPLICATIONS

WESTINGHOUSE HANFORD COMPANY

RICHLAND, WASHINGTON

a 
WHC-SD-TD-OMM-002, REV. 0

TABLE OF CONTENTS

\section{OPERATION AND MAINTENANCE MANUAL \\ FOR THE HIGH RESOLUTION STEREOSCOPIC VIDEO CAMERA SYSTEM \\ (HRSVS) \\ SYSTEM 6230}

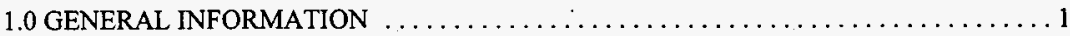

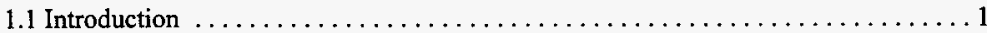

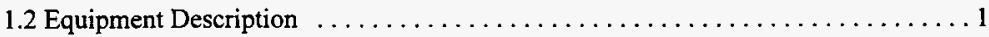

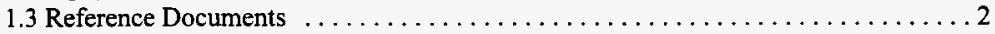

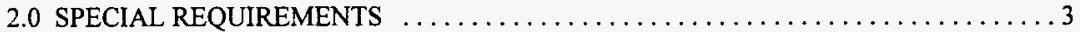

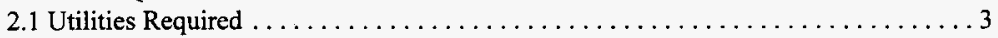

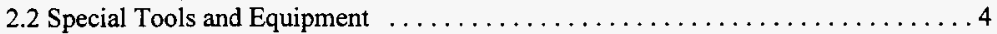

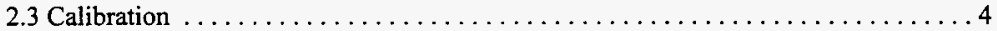

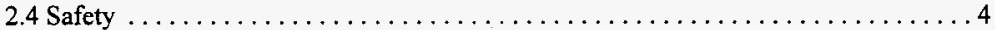

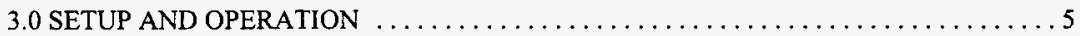

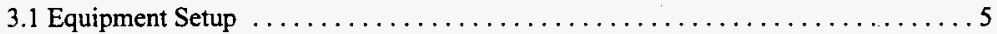

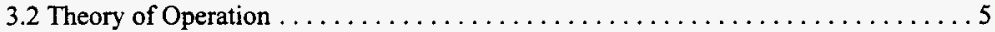

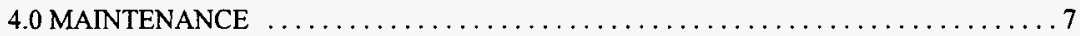

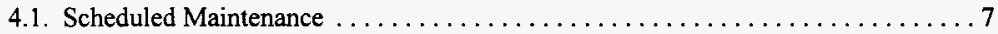

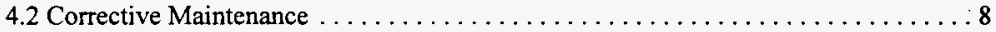

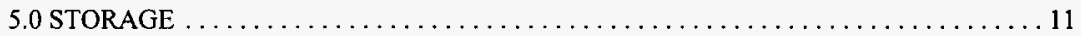

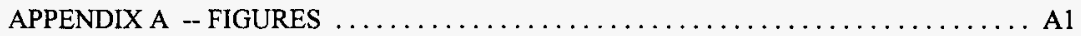

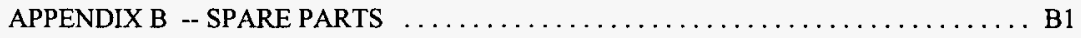

APPENDIX C - HRSVS CALIBRATION $\ldots \ldots \ldots \ldots \ldots \ldots \ldots \ldots \ldots \ldots, \ldots \ldots \ldots \ldots$ 
WHC-SD-TD-OMM-002, REV. 0

\section{TRADEMARKS}

$\mathrm{KEPCO}$ is a trademark of Kepko Inc.

Hypertronics is a trademark of Hypertronics Corporation Dow Corning is a trademark of Dow Corning Corporation Hardigg is a trademark of Hardigg Inc. 
WHC-SD-TD-OMM-002, REV. 0

\section{OPERATION AND MAINTENANCE MANUAL FOR THE HIGH RESOLUTION STEREOSCOPIC VIDEO CAMERA SYSTEM (HRSVS)}

This document defines the requirements for the operation, maintenance, and storage of the High Resolution Stereoscopic Video Camera System (frequently referred to as the Closeup Video Camera System) used with the Light Duty Utility Arm (LDUA) system.

\subsection{GENERAL INFORMATION}

\subsection{Introduction}

The High Resolution Stereoscopic Video Camera System, system 6230, (HRSVS) is a stereoscopic camera system that will be used as an end effector on the LDUA to perform surveillance and inspection activities within Hanford waste tanks (Figure 1). It is attached to the LDUA by means of a Tool Interface Plate (TIP) (Figure 2) which provides a feed through for all electrical and pneumatic utilities needed by the end effector to operate.

\subsection{Equipment Description}

The system consists of the HRSVS end effector and associated support electronics (located in the At Tank Instrument Inclosure (ATIE)). The end effector includes 2 black and white cameras located near the front of the end effector. The purpose of these cameras is to provide general tank feature observations from a distance and to assist the LDUA in maneuvering close to in-tank structures for closer viewing using the stereoscopic cameras (Figure 3).

Located within the end effector are two color cameras (Figure 3 ) and a lens turret assembly (Figure 4). This combination of color cameras and lenses provide closeup 3-D viewing of tank features such as corrosion and tank wall pitting.

All camera functions are controlled using the LDUA Supervisory Data Acquisition System (SDAS).

In the event of a low purge pressure, a signal from the LDUA control system will be provided to the end effector support electronics to instigate a shutdown of all power to the end effector. 


\section{WHC-SD-TD-OMM-002, REV. 0}

\subsection{Reference Documents}

The documents listed may be consulted as a source of information for the basic requirements of the LDUA High Resolution Stereoscopic Video Camera System (sometimes referred to as the close-up stereo video system (CSVS).

$$
\begin{array}{ll}
\text { WHC-SD-TD-CR-001, Rev. } 1 & \begin{array}{l}
\text { Design Criteria for the Light Duty Utility } \\
\text { Arm System End Effectors }
\end{array} \\
\text { WHC-SD-TD-FRD-003, Rev. 0 } & \begin{array}{l}
\text { Functions and Requirements for the Light } \\
\text { Duty Utility Arm Integrated System }
\end{array}
\end{array}
$$

\section{MECHANICAL DRAWINGS}

H-6-14242 SH 1 Rev, 0

(EES-22408-R1-027 Rev. B)

H-6-14242 SH 2 Rev. 0

(EES-22408-R1-027 Rev. B)

H-6-14243 SH 1 Rev. 0

(EES-22408-R4-028 Rev. B)

H-6-14243 SH 2 Rev. 0

(EES-22408-R4-029 Rev. B)

H-6-14243 SH 3 Rev. 0

(EES-22408-R4-031 Rev. B)

H-6-14244 Rev. 0

(EES-22408-R4-030 Rev. B)

H-6-14245 Rev. 0

(EES-22408-R3-032 Rev. B)

\section{ELECTRICAL DRAWINGS}

H-6-14246 Rev. 0

(EES-22408-L7-048 Rev. B)
Close-Up Video System Assembly

Close-Up Video System Assembly

Close-Up Video System Details - Sheet 1

Close-Up Video System Details - Sheet 2

Close-Up Video System Details - Sheet 3

Close-Up Video System Camera Housing Weldment

Close-Up Video System Light Baffle Weldment and Misc Details
UST-ID Close-Up Stereo Camera Housing Schematic 
WHC-SD-TD-OMM-002, REV. 0

CVEE CHASSIS AND POWER SUPPY CHASSIS DRAWINGS

CVEE CHASSIS

H-6-14270 Rev. A Sheet 1 of 9 Common Video End Effector (CVEE) Support Electronics

H-6-14270 Rev. A Sheet 3 of 9 CVEE and HRSVS end to end drawing

H-6-14270 Rev. A Sheet 4 of 9 Common Video End Effector (CVEE) Chassis Layout

H-6-14270 Rev. A Sheet 5 of 9 CVEE Chassis Preparation

H-6-14270 Rev. A Sheet 6 of 9 CVEE Chassis Wiring Diagram (Part A)

H-6-14270 Rev. A Sheet 7 of 9 CVEE Wiring Diagram (Part B)

H-6-14270 Rev. A Sheet 8 of 9 CVEE/Kepco' Interface

H-6-14270 Rev. A Sheet 9 of 9 CVEE/PC Serial Connections

POWER SUPPLY DRAWINGS

H-6-14271 Rev. A Sheet 1 of 3 WHC Power Chassis Layout (CVEE)

H-6-14271 Rev. A Sheet 2 of 3 WHC Power Chassis Wiring Diagram (CVEE)

H-6-14271 Rev. A Sheet 3 of 3 WHC Power Chassis Schematic (CVEE)

\subsection{SPECIAL REQUIREMENTS}

\subsection{Utilities Required}

The HRSVS utilities are supplied to the end effector by the support electronics, more commonly known as the Common Video End Effector (CVEE) support electronics (H-6-14270 \& H-6-14271). The CVEE is located within the ATIE and is connected to the actual HRSVS end 
effector utilizing the ATIE cabling and the umbilical located within the LDUA. The CVEE support electronics consist of the CVEE chassis containing the opto-22 modules and the power supply chassis containing power supplies for camera, motors, and lights. The power requirement for the CVEE and power supply chassis is one each, $120 \mathrm{Vac} / 60 \mathrm{~Hz}$, single phase.

\subsection{Special Tools and Equipment}

Handling of the HRSVS is accomplished using the LDUA Manual End Effector Exchange System (MEEES). This system performs all the manual lifting and engaging of the HRSVS to the LDUA.

\subsection{Calibration}

Calibration of the HRSVS is not readily performed in field applications. A calibration target has been fabricated and installed in the FMEF test facility and is the preferred calibration assembly for the HRSVS. Calibration will only be required when specific HRSVS components (i.e. camera or lenses) are replaced or if the HRSVS is damaged(i.e. camera or lenses are knocked out of position). Provisions for field calibration are being considered. Calibration of the HRSVS can be performed by operations personnel using the LDUA target assembly. This calibration is more of a visual alignment and focusing technique. Appendix $\mathrm{C}$ provides a calibration technique for camera alignment and focusing of the HRSVS.

\subsection{Safety}

The following safety considerations shall be considered when operating and maintaining the HRSVS system.

\subsubsection{Personnel Precautions}

- All operation and maintenance shall be performed in accordance with applicable safety requirements as stipulated in WHC safety documents.

- Before beginning a task, the Person-In-Charge (PIC) is responsible to assure that all personnel taking part in the operation of the HRSVS have been briefed on it operation and that they understand the task being performed and any hazards associated with it.

\subsubsection{Equipment Precautions}

- $\quad{ }^{*}$ CAUTION** The exterior of the HRSVS gets thermally hot when the lights are left on for a time. Use gloves when working with hot surfaces. 
WHC-SD-TD-OMM-002, REV. 0

- Avoid contact with the 120 Vac power connections on connection strips.

\subsection{SETUP AND OPERATION}

This section provides instructions for the normal handling and operation of the HRSVS in support of routine operations of the LDUA within a Hanford tank.

\subsection{Equipment Setup}

3.1.1 The HRSVS will be stored in a plastic bag within the hard plastic storage container. The top section of the storage container will be removed exposing the top of the end effector. Once the plastic bag is removed the end effector will be available for removal from the storage container using the MEEES.

3.1.2 The door of the TRIC will be opened and the MEEES will place the HRSVS in position for connection to the LDUA. This is accomplished using the TIP connecting mechanism.

3.1.3 Under normal operation the CVEE chassis and CVEE power supply chassis will be energized and all cable connections between the CVEE chassis and the LDUA interface will be made. Therefore once the HRSVS end effector is placed on the arm the system will be ready for operation. Actual operation of the HRSVS is performed utilizing SDAS. Figure 5 is the SDAS Virtual Instrument (VI) that will be used to remotely operate the HRSVS.

3.1.4 Using the VI the operator can turn on pairs of lights for improved viewing. The two camera systems can be interchanged for general purpose viewing, or closeup $3-\mathrm{D}$ viewing. The lens turret assembly can be rotated for various magnifications of the closeup capability. The large focusing lens can be adjusted to provide precise focus of the viewed object. The convergence of the two color cameras can also be adjusted for a more comfortable stereo effect.

\subsection{Theory of Operation}

3.2.1 Once the HRSVS is placed on the LDUA it will be lowered into the waste tank. The black and white cameras will be used first to provide an overview of the internal features of the waste tank. Each of these cameras have a different field of view. The left camera (from camera view) has a $6 \mathrm{~mm}$ lens. The right camera has a $3.7 \mathrm{~mm}$ lens which provides a little wider field of view. The black and white 


\section{WHC-SD-TD-OMM-002, REV. 0}

cameras a low lux cameras and should not require an excessive amount of light for proper operation. The LDUA will provide general movement of the end effector within the waste tank. The black and white camera can be used to pick out interesting in-tank features that the operator can then look at using the closeup system.

3.2.2 Once a feature has been picked out, the black and white cameras will provide enough visual information for the LDUA operator to maneuver the LDUA within approximately 3 feet of the feature to be observed. The operator can then switch to the color stereo camera pair and look at the feature using the stereoscopic system. The LDUA will be used for gross focusing by moving the HRSVS closer to or away from the feature. Once the feature is relative in focus, the HRSVS turret and lenses can be adjusted for optimum viewing, along with the large focusing lens, and camera convergence. Some precise vertical and horizontal image adjustments can be made by the stereo system located in the Operations Control Trailer (OCT) along with some color and contrast enhancement.

3.2.5 After the HRSVS has performed it task of surveillance and inspection, it will be raised out of the tank and removed from the LDUA, and other end effectors can now be used. 


\section{WHC-SD-TD-OMM-002, REV. 0}

\subsection{MAINTENANCE}

\subsection{Scheduled Maintenance}

Scheduled maintenance is that preventive maintenance that is performed on the HRSVS at regular time intervals. The PIC shall be responsible for assuring that the designated maintenance activities are accomplished at the appropriate time interval as specified below.

Unless specifically directed otherwise, all maintenance shall be done with the HRSVS and support electronics disconnected from the power source.

4.1.1 Daily Maintenance (i.e. each additional 8 hours of operating time)

- Using a soft cloth and commercial window cleaner, gently clean all optical windows.

- Perform a general visual inspection.

4.1.2 Weekly Maintenance (i.e. each additional 40 hours of operating time)

- Perform a visual inspection to make sure all hex screws are fully backed out into the outer shell of the HRSVS.

4.1.3 Six-Month Maintenance (i.e. each additional 172 hours of operating time)

- Replace all accessible o-rings (no need to replace o-rings that have been previously sealed using silicon sealants).

- Perform a general visual inspection of the interior of the HRSVS end effector.

- Perform a general visual inspection of the CVEE chassis and power supply chassis.

4.1.4 Twelve-Month Maintenance (i.e. each additional 344 hours of operating time)

- Replace camera and lens assemblies (will require recalibration).

- Replace all lamps. 


\subsection{Corrective Maintenance}

Corrective maintenance addresses those situations where remedy is required to correct component failures which occur unexpectedly, and are therefore not performed in accordance with a schedule. Rigorous adherence to scheduled preventive maintenance should minimize the need to perform corrective maintenance. The components in the HRSVS are expected to have a reasonably long operating life, and corrective maintenance activities are minimal. The following items have been identified as potential corrective maintenance activities. A recommended spare parts list has been included in Appendix A. A complete parts list can be found on the HRSVS assembly drawing. Component original equipment manufacturer (OEM) documentation can be found in the HRSVS subsystem component specification file. The OEM documentation should provide sufficient information to assist in the adjustments or maintenance activities for the specific component.

Performance of unscheduled maintenance may require access to the LDUA SDAS and associated equipment for system checkout. Unscheduled maintenance may also require a recalibration of the HRSVS which may require sufficient decontamination to allow calibration in a non-radiation zone.

1) Lamp replacement (note: to replace the lamps care must be taken not to touch the halogen bulb with bare hands. Use rubber or cotton gloves to handle bulb and reflector assembly).

a. Unscrew the cover plate assembly (Figure 6) and remove.

b. Remove each bulb carefully and replace by inserting new bulb. Make sure new bulb is seated fully into its socket.

c. Reinstall the cover plate.

d. Power light to verify proper operation.

2) Camera and Lens replacement

a. Screw inward on the hex screws located at the end of the end effector (away from TIP).

b. Carefully push the interior HRSVS assembly out of the stainless steel shell (Figure 6). Note: the sections of the HRSVS have been marked so that they can be easily reassembled.

c. Disconnect the black and white camera wiring.

d. Remove screws holding the black and white cameras on the $\mathrm{L}$ shaped bracket.

e. Replace the camera, and lens assembly in the same position as the one removed.. 
WHC-SD-TD-OMM-002, REV. 0

f. Reconnect camera wiring.

g. Disconnect the color camera wiring. Remove each color camera by removing fastening screws in the covering bracket. Cameras will then slide out of the $U$ shaped brackets. Note that these cameras have been shimmed for proper rotational alignment.

h. Replace the color cameras in the same position as the ones removed. Note, these cameras will need to be properly aligned both in the radial direction and in rotation. This is done using the alignment target in Appendix B.

I. Reconnect camera wiring.

j. Carefully reassemble the HRSVS.

4) Base O-ring Replacement

All o-rings on the HRSVS can be worn or damaged during assembly and disassembly. To replace the o-ring which is at the interface between the HRSVS and the TIP it will be necessary to remove the TIP (Figure 7). All other o-rings can be replaced by disassembling the HRSVS as in step 3 above and using the same clean and grease technique as below.

a. Position the HRSVS/TIP on its side.

b. Remove the TIP from the HRSVS by removing the six bolts in the TIP. Reference SPAR documentation for the bolt location in the TIP. The bolts will require a 5/16" hex head driver. After the bolts are removed, carefully separate the TIP from the HRSVS, taking care not to strain the Hypertronic ${ }^{2}$ connectors. These connectors will need to be unscrewed and pushed through their mounting holes.

c. Remove the o-ring from the groove. Clean the o-ring groove and reapply Dow Corning ${ }^{3}$ High Vacuum grease or equivalent to the o-ring.

d. Replace the o-ring until it is positioned in the groove.

e. Reinstall the TIP to the HRSVS. There are two alignment dowels in the TIP which will align the TIP to the HRSVS. Carefully align the TIP with the HRSVS so that the two pieces mate properly and the o-ring is not pinched. Reinstall the six bolts in the TIP to secure the TIP to the HRSVS.

${ }^{2} \mathrm{Hypertronics}$ is a trademark of Hypertronics Corp.

${ }^{3}$ Dow Corning is a trademark of Dow Corning Corp. 
5) Optical window replacement (note: all optical windows have been installed using an ultraviolet cured cement).

a. To replace windows, the end plate of the HRSVS must be disassembled and the window knocked out using a blunt instrument and all remaining window material removed. A new cut-to-fit window may then be reinstalled and glued in place.

b. Replace the end plate on the HRSVS. 


\section{WHC-SD-TD-OMM-002, REV. 0}

\subsection{STORAGE}

During normal operation the of the LDUA system, the HRSVS will reside in its storage container until it is needed for surveillance and alignment activities. This storage container is a large molded plastic container (Hardigg ${ }^{4}$, case molded plastic) with internal shock aborbing material (polyethylene (closed cell) foam). The HRSVS will be placed in a plastic bag after the decontamination process and sealed shut. It will then be placed into the storage container and the top of the container fastened. The storage container will be stored in the LDUA lay down area until needed. 
WHC-SD-TD-OMM-002, REV. 0

APPENDIX A -- FIGURES 
WHC-SD-TD-OMM-002, REV. 0

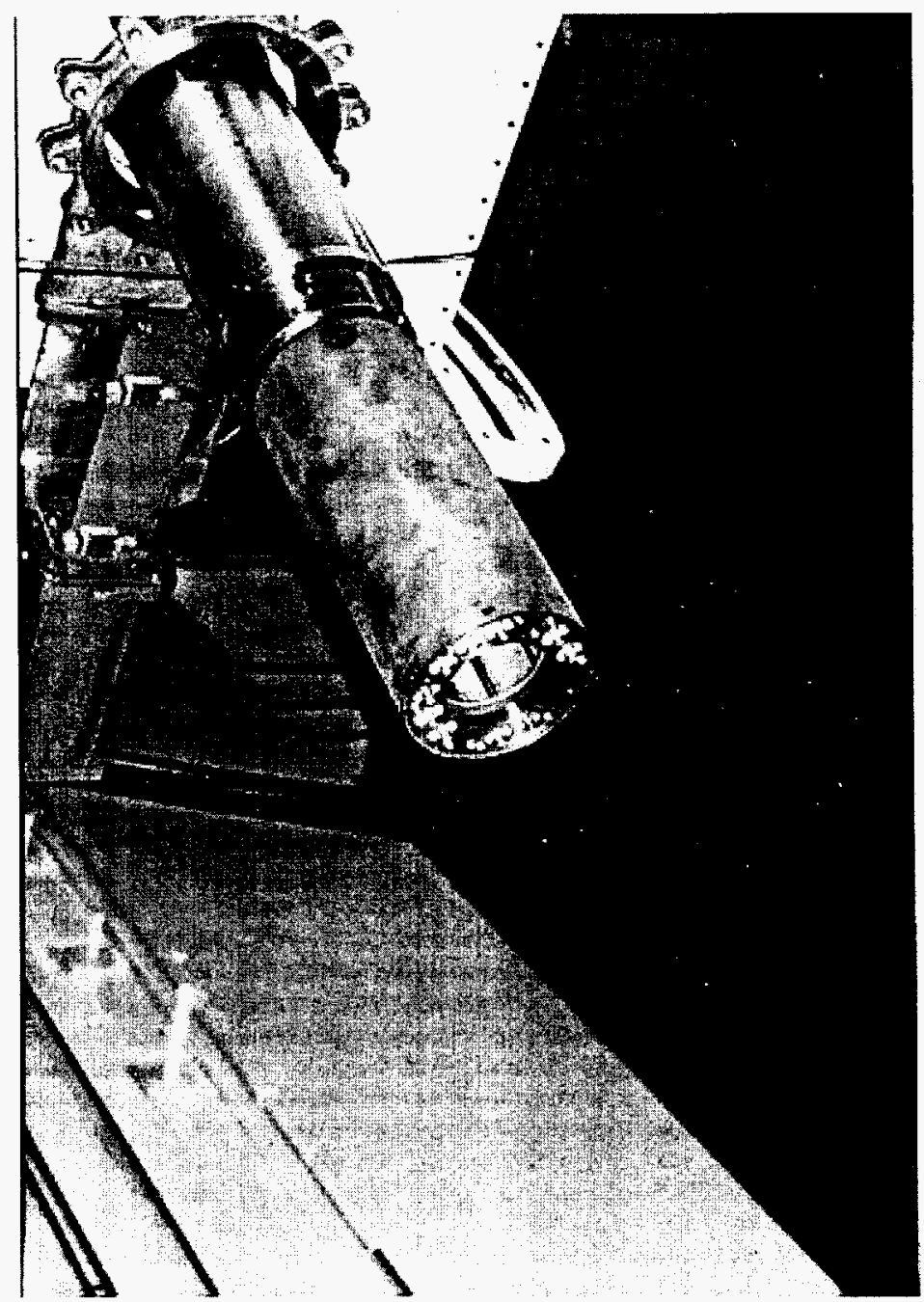

Figure 1 HRSVS mounted on the end of the LDUA 
WHC-SD-TD-OMM-002, REV. 0

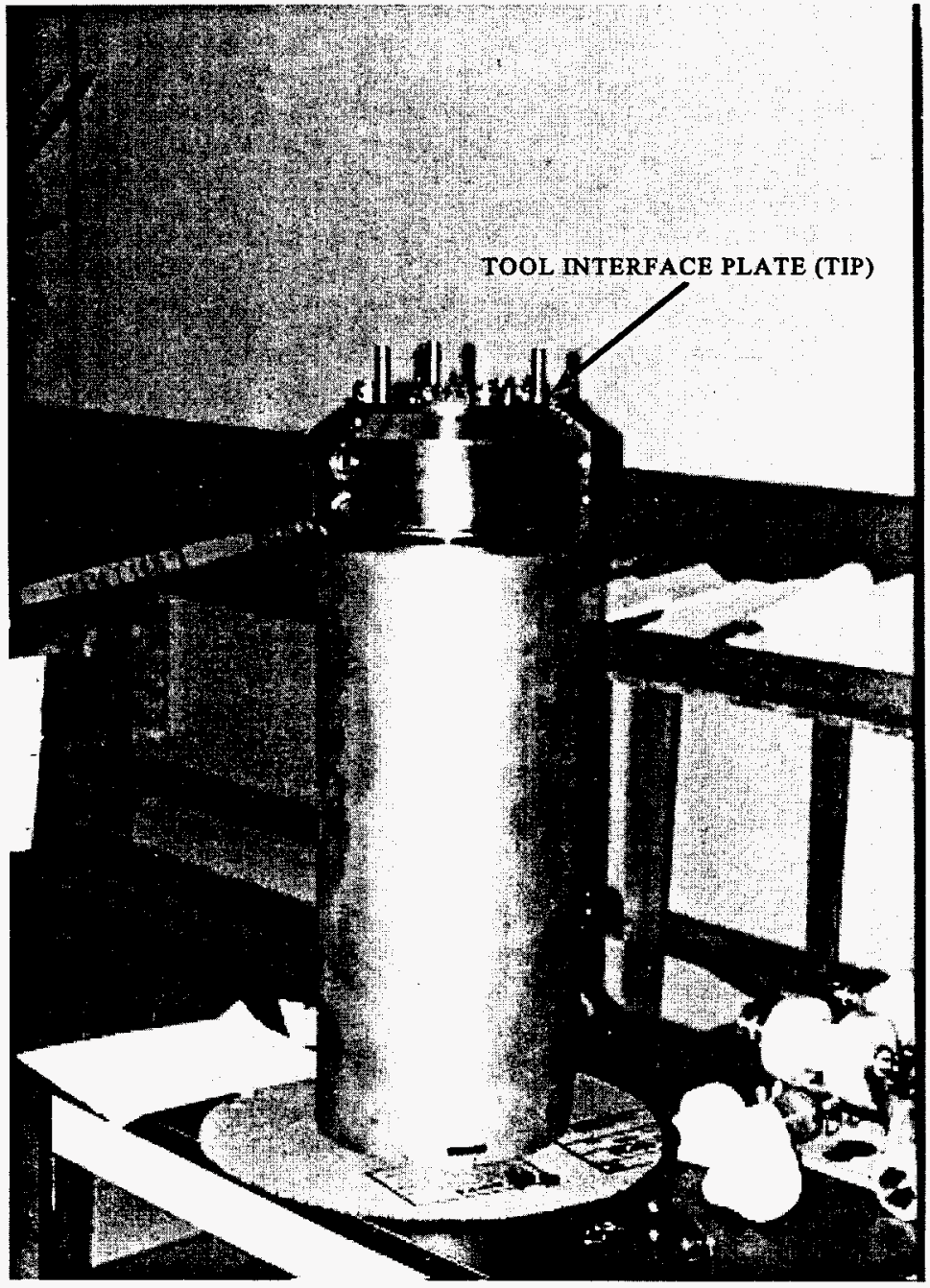

Figure 2 Tool Interface Plate (TIP) Attached to the HRSVS 
WHC-SD-TD-OMM-002, REV. 0

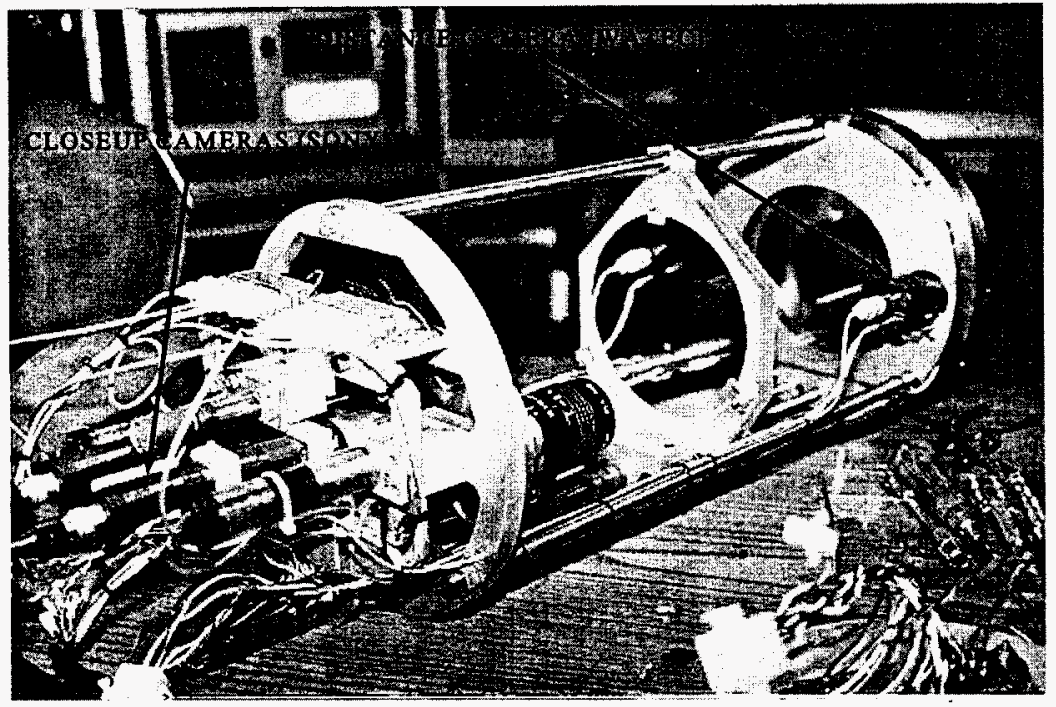

Figure 3 Cameras Used in the HRSVS 
WHC-SD-TD-OMM-002, REV. 0

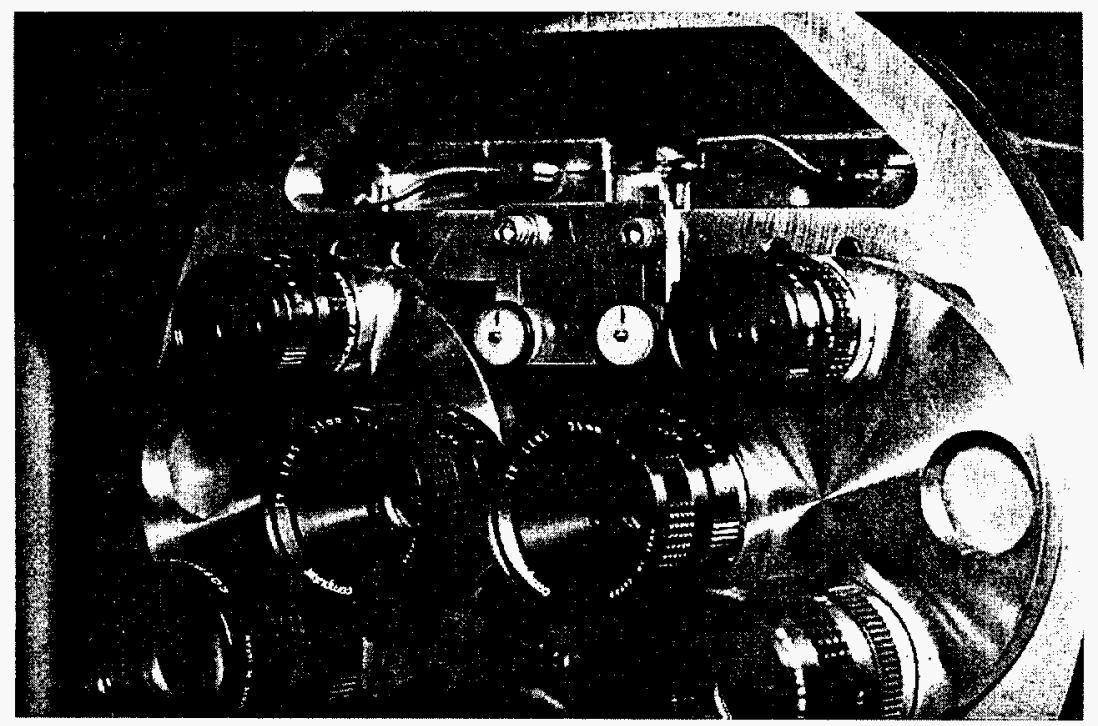

Figure 4 Lens and Turret Assembly 
WHC-SD-TD-OMM-002, REV. 0

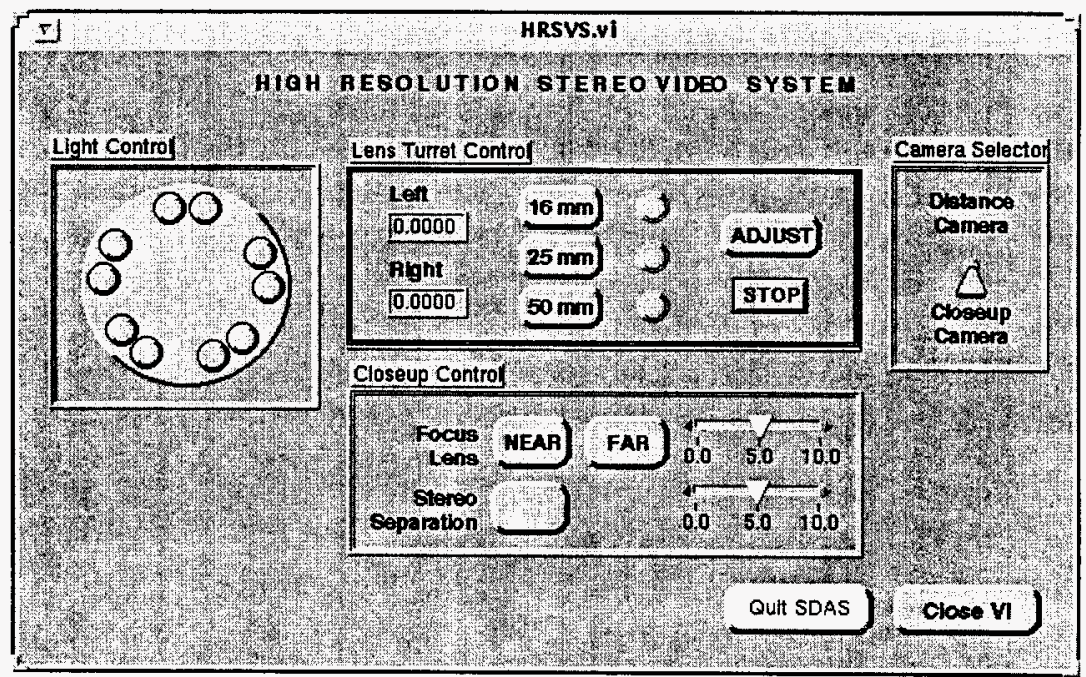

Figure 5 HRSVS Virtual Instrument (VI) 
WHC-SD-TD-OMM-002, REV. 0

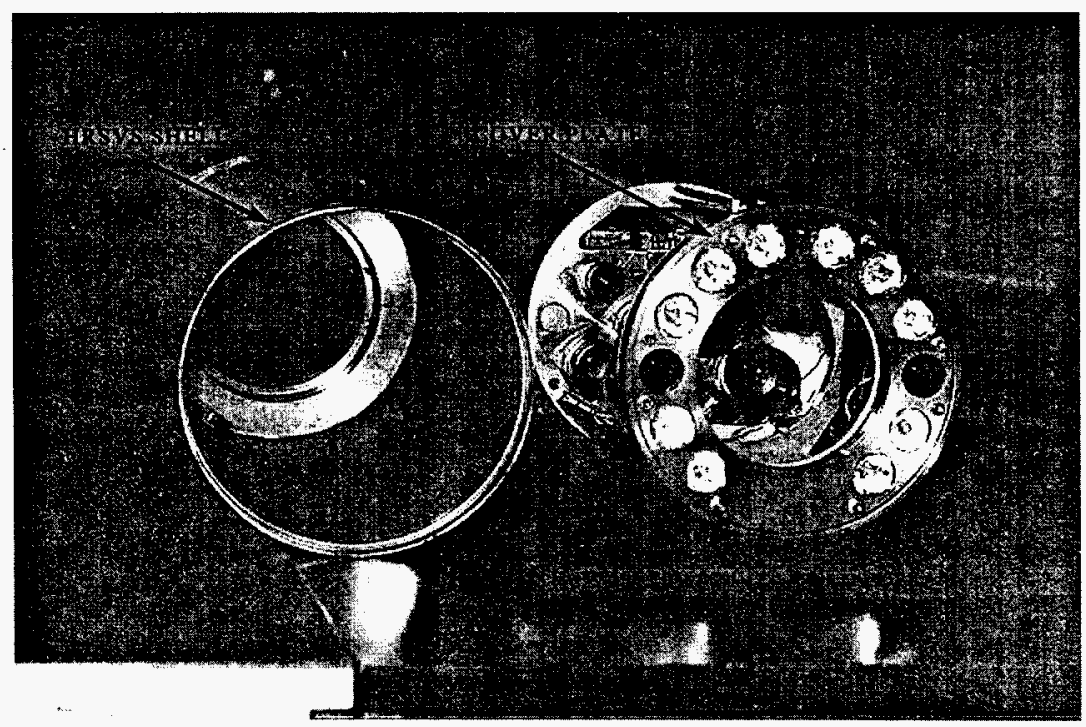

Figure 6 HRSVS Removed from its Shell 
WHC-SD-TD-OMM-002, REV. 0

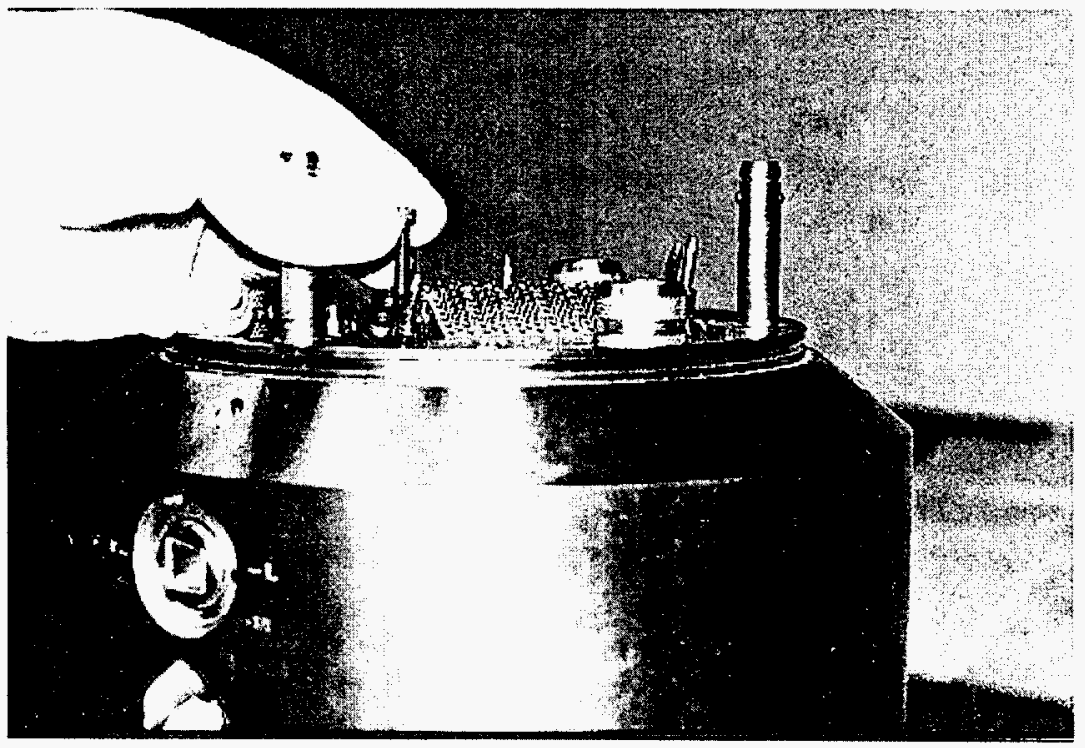

Figure 7 Removing the TIP from the HRSVS 


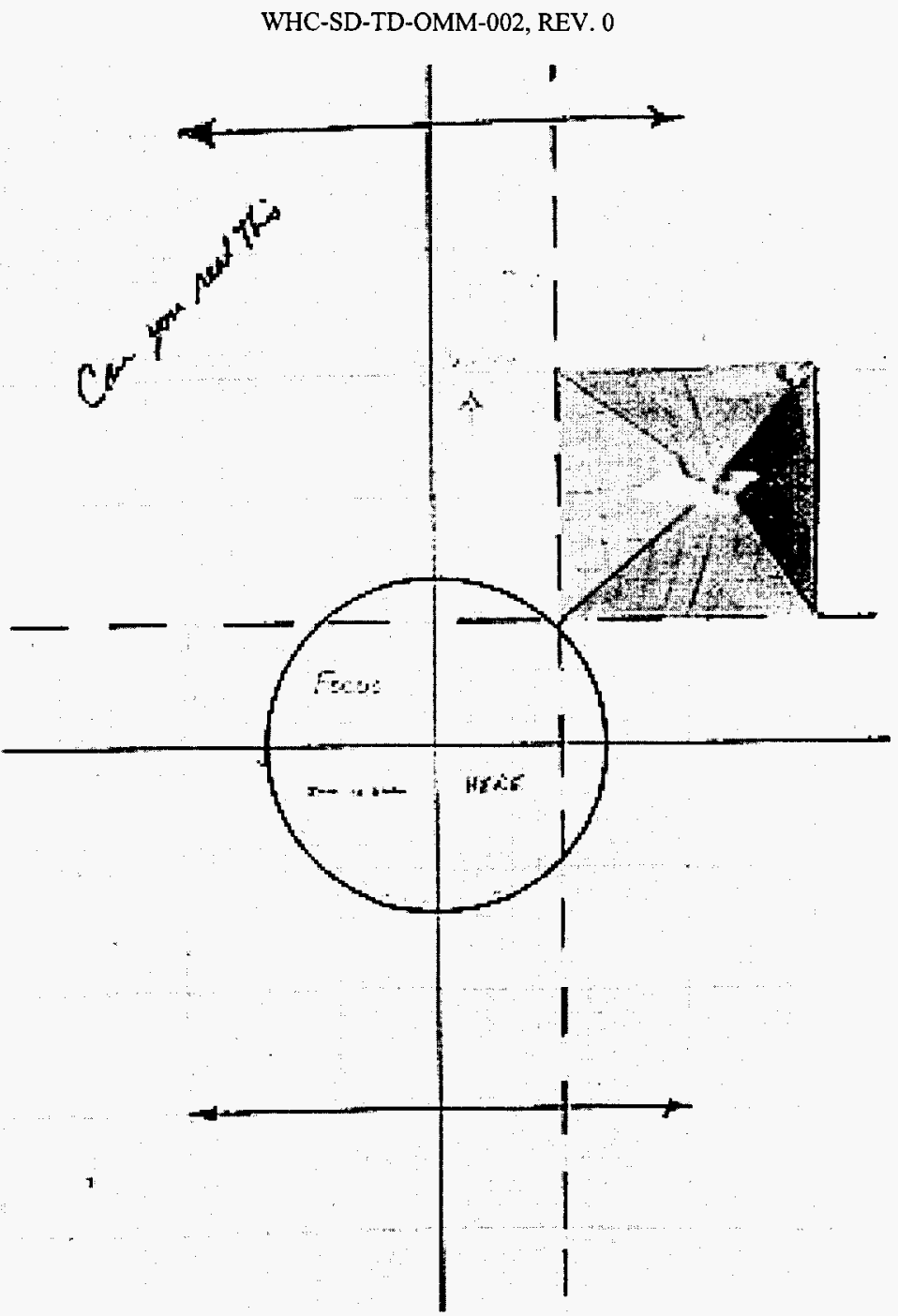

Figure 8 HRSVS Alignment Target 
WHC-SD-TD-OMM-002, REV. 0

APPENDIX B -- SPARE PARTS

B1 


\section{WHC-SD-TD-OMM-002, REV. 0}

The following is a recommended OEM spare parts list for the HRSVS end effector.

1) Video Camera, Sony, Model \#XC-999

2) Black and White Camera, Watec, Model \#902A.

3) Lens, $16 \mathrm{~mm}$, Cosmicar

4) Lens, $25 \mathrm{~mm}$, Cosmicar

5) Lens, 50mm, Computar

6) Lens, $3.7 \mathrm{~mm}$, Cosmicar

7) Lens, $6 \mathrm{~mm}$, Cosmicar

8) Halogen Lamp, Ostam, Model \#35MR16/18/SP

9) Halogen Lamp, Osram, Model \#35MR16/38/FL

10) O-Ring, 10.00 DIA X 1/8 THK, Ethylene Propylene, Parker \#2-273 E540-80

11) O-Ring, 1.38 DIA X 3/32 THK, Ethylene Propylene, Parker \#2-123 E540-80

12) O-Ring, 9.50 DIA X 1/8 THK, Ethylene Propylene, Parker \#2-271 E540-80 
WHC-SD-TD-OMM-002, REV. 0

\section{APPENDIX C -- HRSVS CALIBRATION}


WHC-SD-TD-OMM-002, REV. 0

Calibration of the HRSVS can be performed using the HRSVS test target (Figure 8) and test fixtures located in the FMEF facility. The procedure for calibration is as follows:

1) Remove the outer shell from the HRSVS assembly and place the HRSVS on a table using nylon test rollers so it can be rotated.

2) Connect the HRSVS to the LDUA SDAS using the LDUA test umbilical and power on the CVEE chassis. The HRSVS should become operational.

3) Level the HRSVS in the long axis direction. Level the HRSVS in the radial direction by rotating the HRSVS until the two Sony cameras are level.

4) Black and White Camera Adjustments (Distance Cameras)

4a) Use a coaxial Tee to provide video to a regular video monitor located near the HRSVS.

4b) Instruct the SDAS operator to switch to the Distance cameras.

4c) Manually operate the focus on the camera until video is in focus.

4d) Manually operate the iris on the camera until video is bright and clear.

4e) Match the same characteristics to the other black and white camera.

4f) Optional: You may use a five minute epoxy to affix the lens adjustments to maintain their position so that they cannot be altered.

5) Color Camera Adjustments (Closeup Cameras)

5a) Use a coaxial Tee to provide video from each of the two Sony cameras to the Stereographics 3-D system (Stereographics view/record box, infrared emitter, and stereo ready monitor must be powered on. It is preferred that this stereo system be in close proximity to the HRSVS during calibration). The left camera must plug into the left camera input on the view/record box. The right camera must plug into the right camera input.

5b) Instruct the SDAS operator to switch to the Closeup cameras.

5c) Instruct the SDAS operator to rotate the lens turrets to the $50 \mathrm{~mm}$ lens 
WHC-SD-TD-OMM-002, REV. 0

position.

5d) Instruct the SDAS operator to position the large focusing lens to approximately the midpoint position of its range.

5e) Position the test target approximately 38 inches in front of the large focusing lens, centered with the end effector.

5f) Instruct the SDAS operator to turn on the pair of lights at the top of the HRSVS.

5g) Adjust each lenses focus and iris so that each image is clear and with proper brightness. Each camera can be viewed separately by either covering one of the camera lenses or disconnecting the coax to that specific camera.

5h) Instruct the SDAS operator to rotate to each lens pair and repeat the lens adjustments.

5i) Instruct the SDAS operator to return the lens arrangement to the $50 \mathrm{~mm}$ lens pair.

5j) Using the Stereographics system align the horizontal lines of the test target on both video images. This can be tedious process. It will require thin shim stock to place under portions of the cameras to provide a rotation. This rotation will provide the ability to align the horizontal line of the test target.

5k) Optional: Once all the lenses are adjusted they may be fixed using epoxy.

51) Power down the CVEE equipment and disconnect the LDUA umbilical. The HRSVS can now be reassembled. 